\title{
Multi-wavelength spatially resolved analysis of quasi-periodic pulsations in a solar flare
}

\author{
A. R. Inglis ${ }^{1}$, V. M. Nakariakov ${ }^{1}$, and V. F. Melnikov ${ }^{2}$ \\ 1 Centre for Fusion, Space and Astrophysics, Physics Department, University of Warwick, Coventry, CV4 7AL, UK \\ 2 Radiophysical Research Institute (NIRFI), Nizhny Novgorod 603950, Russia
}

Received 23 December 2007 / Accepted 12 June 2008

ABSTRACT

\begin{abstract}
Aims. We aim to perform a spatially resolved analysis of a quasi-periodic pulsation event from 8th May 1998 using microwave data from the Nobeyama Radioheliograph and Radiopolarimeter, and X-ray data from the Yohkoh satellite.

Methods. Time spectra of the signals integrated over the emission source are constructed with the use of the Lomb-Scargle periodogram method, revealing the presence of a pronounced 16 s periodicity. The Pixon image reconstruction algorithm and Hanaoka algorithm are used to reconstruct images from the hard X-ray data from Yohkoh/HXT and Nobeyama Radioheliograph respectively. The phase relationship of the microwave emission was analysed with the use of cross-correlation techniques.

Results. The flaring loop was resolved in the microwave band. The hard X-ray sources are found to be located near the footpoint and at the loop apex determined by the soft X-ray image. The apex source is much fainter than footpoint one. In microwave, all parts of the loop are seen to oscillate with the same period and almost in phase. It was not possible to determine the spatial structure of the oscillation in the hard X-ray band. The period and the coherent spatial structure of the oscillation are indicative of the presence of either an MHD sausage mode or a periodic regime of magnetic reconnection.
\end{abstract}

Key words. Sun: flares - Sun: oscillations - waves - Sun: corona - magnetohydrodynamics (MHD)

\section{Introduction}

Quasi-periodic pulsations (QPP) have been seen in emission from solar flares for several decades (Nakariakov 2007). The emission intensity from a solar flare is observed to oscillate with a characteristic period, sometimes stably for several minutes, other times in short bursts. The oscillation periods have been observed to vary from a few seconds up to several minutes. In some cases oscillations with more than one characteristic period occur simultaneously (Melnikov et al. 2005). Presently, the interest in flaring QPP is connected mainly with the development of coronal seismological techniques for determination of physical conditions and processes operating in flaring active regions. Also, the understanding of flaring QPP in the solar corona will open up very interesting perspectives for the diagnostics of stellar coronae (Mathioudakis et al. 2003; Mitra-Kraev et al. 2005). In the stellar context, coronal seismological methods are able to give additional constraints on the physical parameters, compensating for the lack of spatial resolution (Nakariakov 2007).

Various suggestions have been made regarding possible causes of QPP in solar flares. One possible mechanism is the modulation of physical parameters of the emitting plasma by magnetohydrodynamic (MHD) modes of the flaring plasma structure. All four kinds of MHD modes can produce modulation: torsional (Tapping 1983), longitudinal (Nakariakov \& Melnikov 2006), kink (Grechnev et al. 2003) and sausage (Nakariakov et al. 2003). In addition, sausage modes can modulate the precipitation rate of the non-thermal electrons accelerated by the flare via periodic change of the magnetic mirror ratio (Zaitsev \& Stepanov 1982). Also, recently (Nakariakov et al. 2006), it has been suggested that QPP in a solar flare might be caused by MHD oscillations in another loop situated near the flare site but magnetically disconnected from the flaring region. The fast waves leaking from the oscillating loop can reach the flaring site and periodically trigger the process of magnetic reconnection. This mechanism can explain the observed periodic variation of the flaring emission in terms of the periodic acceleration of non-thermal electrons. The mechanisms connected with the modulation of the precipitation rate and the periodic triggering of reconnection can explain the observed correlation in time of hard X-ray and microwave QPP.

It has already been emphasized that the correct identification of the mechanism responsible for QPP should be based upon spatially resolved analysis (Grechnev et al. 2003) and (Nakariakov et al. 2003). This analysis includes examination of amplitudes and phase relationships of oscillations in different parts of the flaring loop. In particular, the presence of several different MHD modes with different spatial structure and periods was established in Melnikov et al. (2005). The leading instrument used in these studies is the Nobeyama Radioheliograph (NoRH), which allows one to detect the spatial distribution of the microwave emission of non-thermal electrons, caused by the gyrosynchrotron mechanism, along the flaring loop. In addition, hard X-ray instruments which have comparable spatial and temporal resolution allow one to observe the footpoints of the flaring loop or arcade and sometimes the region above the loop apex.

The aim of this paper is to study QPP in a spatially resolved flaring event, observed simultaneously in microwave and hard $\mathrm{X}$-rays, and to gain insight into the mechanism for the periodic modulation of the emission. The flare discussed here is an M-class event which occurred on the 8th May 1998 on the solar limb. It was observed by the Nobeyama Radioheliograph and the Nobeyama Radiopolarimeter, and by the Yohkoh, SOHO and GOES satellites. This event was first analysed by 

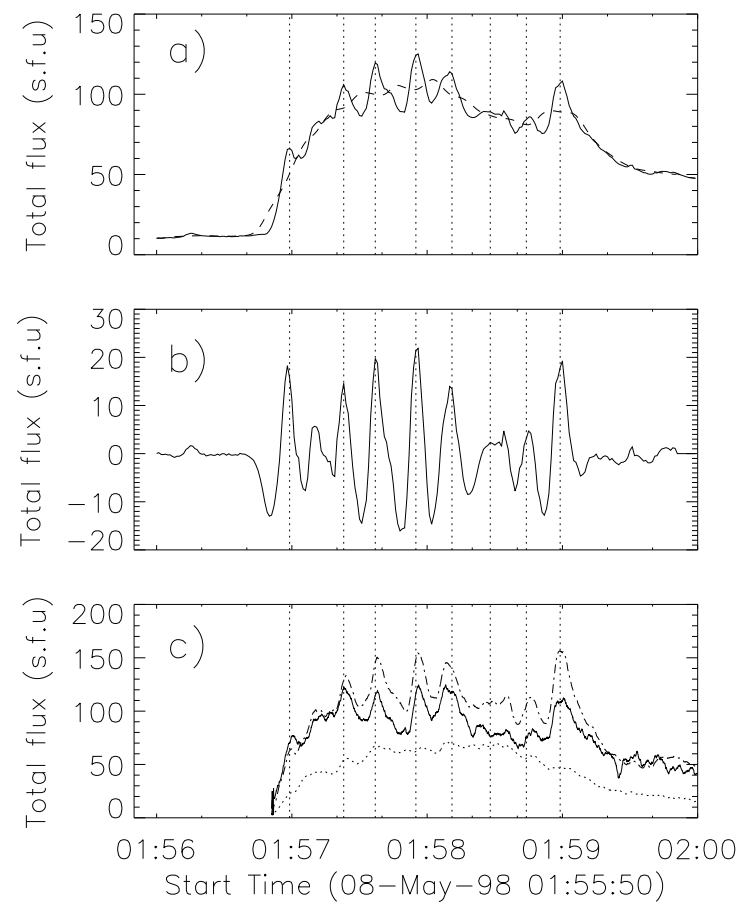

Fig. 1. a) Integrated flux time profile of microwave emission at $17 \mathrm{GHz}$ for the solar flare of 8th May 1998, as observed by NoRH. Overlayed with the dashed line is the background emission profile obtained via $20 \mathrm{~s}$ smoothing of the integrated flux signal. b) The signal after subtraction of the background profile from the integrated flux. c) Solar flux time profiles of microwave emission at $9.4 \mathrm{GHz}$ (dot-dashed line), $17 \mathrm{GHz}$ (solid line) and $3.75 \mathrm{GHz}$ (dotted line), obtained by the Nobeyama Radiopolarimeters.

Stepanov et al. (2004), who found a 16 s periodicity and suggested that it was caused by an MHD ballooning mode. Here, detailed analysis and an alternative interpretation of this event are presented.

\section{Analysis of integrated light curves}

\subsection{Microwave time profiles}

The time profile of the microwave flux integrated over the whole flaring region (the light curve), recorded by NoRH at $17 \mathrm{GHz}$, is shown in Fig. 1. The signal is averaged over $1 \mathrm{~s}$. The time profile shows that this was a single burst event beginning at 01:57 UT. Quasi-harmonic oscillations of large modulation depth are evident immediately after the beginning of the flare. These oscillations do not appear in $34 \mathrm{GHz}$ due to the low signal-to-noise ratio.

The presence of the pronounced periodicity is also seen in the lower frequency channels observed by the Nobeyama Radiopolarimeter (see Fig. 1). The oscillations in different channels are seen to be coincident.

The high quality of the observed oscillation is confirmed by the spectrum of the analysed signal. Subtracting the running mean smoothed by $20 \mathrm{~s}$ from the signal recorded at $17 \mathrm{GHz}$ by NoRH, and applying the periodogram technique described by Scargle (1982) and Horne \& Baliunas (1986), we obtain the power spectrum with the pronounced peak of $16 \mathrm{~s}$ (Fig. 3). The smoothing is equivalent to the removal of the low frequency component of the signal in the spectral domain, which is connected with the long period trend. The observed period concurs
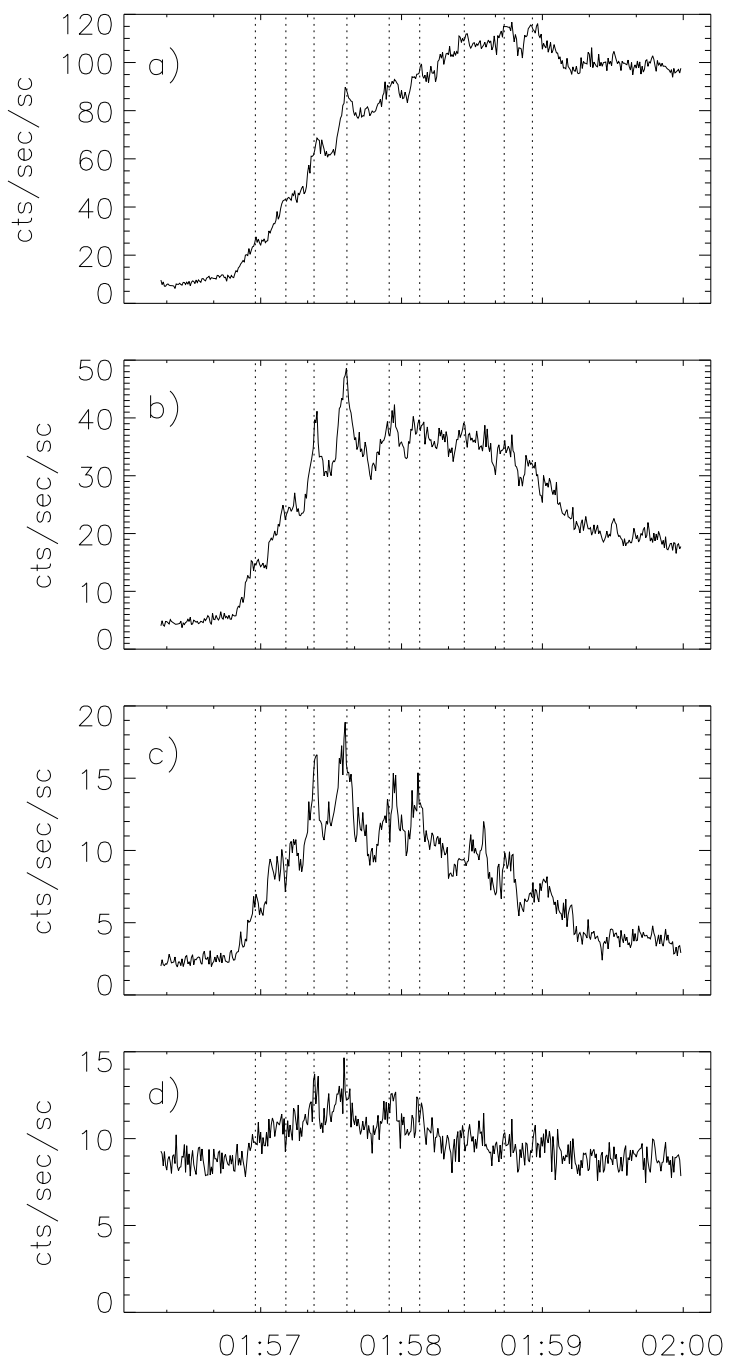

Fig. 2. Hard X-ray light curves from the Yohkoh satellite, observing the solar flare of 8th May 1998. a) X-ray counts in the L (13-23 keV) range, b) X-ray counts in the $\mathrm{M} 1(23-33 \mathrm{keV})$ range, c) $\mathrm{X}$-ray counts in the M2 (33-53 keV) range, d) X-ray counts in the $\mathrm{H}(53-93 \mathrm{keV})$ range.

with the findings of Stepanov et al. (2004). The peak is found to be well above the $99 \%$ confidence level calculated according to Horne \& Baliunas (1986). The main spectral peak is surrounded by secondary peaks. To check whether the secondary peaks appeared because of the finite duration of the sample, we calculated the spectra of the smoothed signals multiplied by window functions (see Fig. 3). This increases the amplitude resolution of the spectrum on the expense of the frequency resolution. According to the figure, the use of a window function clearly suppresses the spectral sidelobes of the peak, indicating that they may be artificial rather than real in nature.

\subsection{Hard X-ray time profiles}

The impulsive burst of emission was also detected by the Hard X-ray Telescope (HXT) on board Yohkoh. Hard X-ray light curves corresponding to different energies are shown in Fig. 2.

There is clear evidence of oscillations in the L, M1 and M2 channels. A periodogram analysis on the modulation depth in hard X-rays reveals a period matching that of the microwave emission above the $90 \%$ confidence level in both the L and M1 

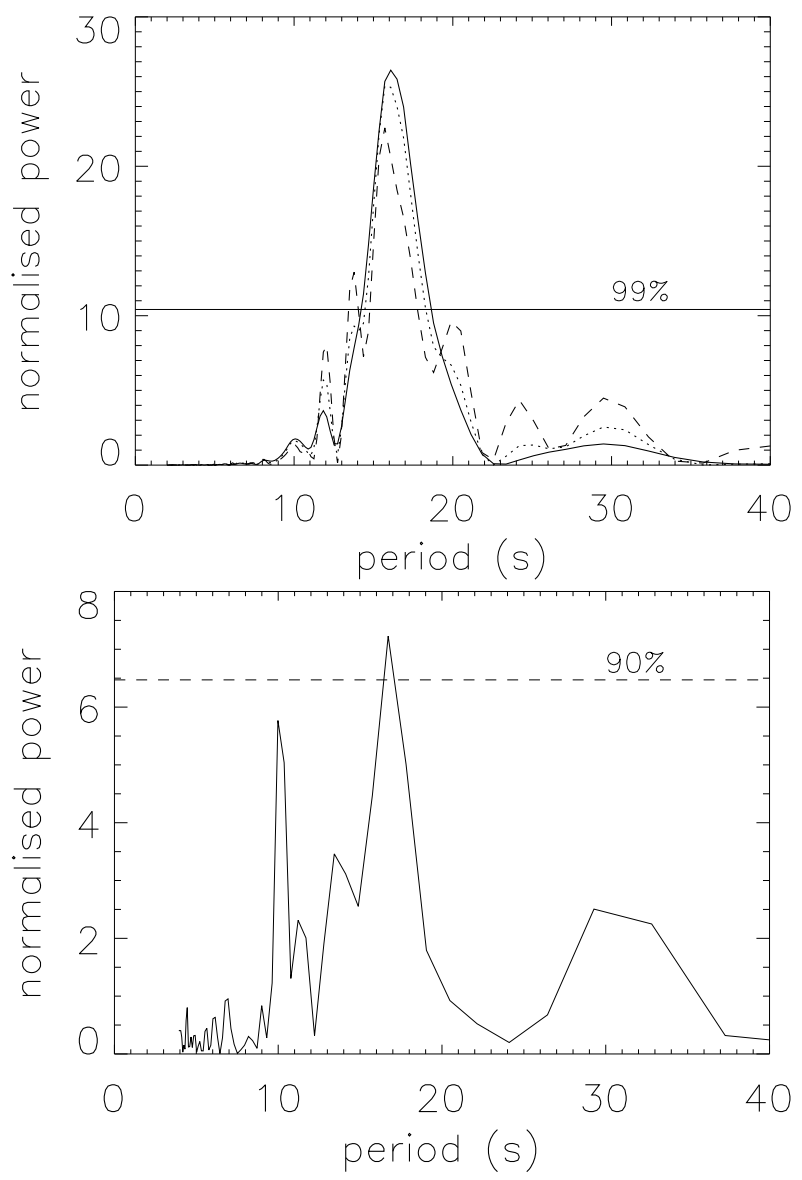

Fig. 3. Top panel: Lomb-Scargle periodogram (Scargle 1982) of the $17 \mathrm{GHz}$ modulation depth from the Nobeyama Radioheliograph. Dashed line: periodogram without a window function; dotted line: periodogram using a Hann window function; solid line: periodogram using a Blackman-Harris window function. The periodogram reveals a oscillation period of $16 \mathrm{~s}$, above the $99 \%$ confidence level. Bottom panel: Lomb-Scargle periodogram of the M1 (23-33 keV) X-ray modulation observed by the Yohkoh satellite.

energy channels. The Scargle periodogram of the oscillation in the M1 channel is shown in Fig. 3. The count rate in the M2 band is too low for a reliable detection, while the $\mathrm{H}$ channel observes little above the background level, consistent with an M-class flaring event.

\section{Locations of emission sources}

Both the NoRH and HXT allow for spatial resolution of the microwave and hard X-ray emission sources, respectively. The location of the various types of emission from a flare have implications for the flare model. According to the standard flare model, magnetic reconnection is supposed to take place above the loop top. Hard X-ray emission is expected at the two footpoints of the flaring loop as a result of the interaction of non-thermal electrons accelerated in the flare with dense plasmas. A third source may also be present above the loop top (Masuda et al. 1994). The source of the microwave emission generated by non-thermal electrons by the gyrosynchrotron mechanism is usually situated at a segment of the loop. Soft X-rays from solar flares are due to thermal emission from the hot plasma which fills the flaring loop. Consequently, soft X-ray images can provide information about the geometry of the flaring region.

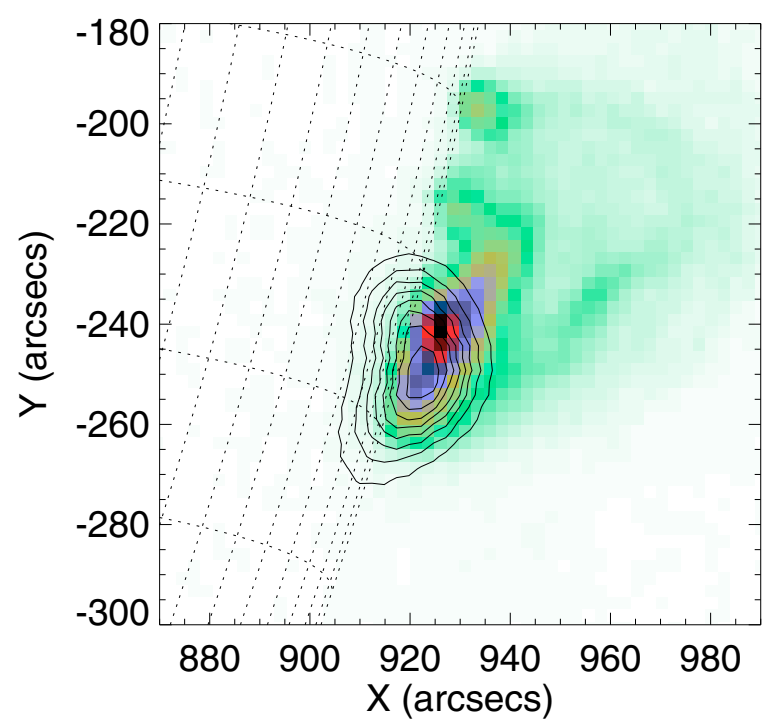

Fig. 4. Image: Soft X-ray image from the Yohkoh satellite of the flare of 8th May 1998. Contour: microwave emission source for the same flare, observed by the Nobeyama Radioheliograph at $17 \mathrm{GHz}$.

A soft X-ray image of the flare provided by the Yohkoh satellite is shown in Fig. 4 with the $17 \mathrm{GHz}$ microwave emission source from the same time overlayed. The microwave emission is seen to take the form of a single source. This source coincides with the maximum emission of the soft X-ray loop, near the southern footpoint. Figure 5 shows the soft X-ray images with the hard X-ray emission sources from the L (13-23 keV), M1 (23-33 kev), and M2 (33-53 kev) channels overlayed. The images indicate that the flare is located on the solar limb. This concurs with the observations made by the Extreme Ultraviolet Imaging Telescope (EIT) on board SOHO, shown in Fig. 6.

The sources of different hard X-ray channels have different structures. The low energy channel emission is similar to the soft $\mathrm{X}$-ray structure and is centred around a single maximum near and a little above the centre of the soft X-ray loop, indicating that this emission is thermal in nature. The higher energy channels however reveal the presence of multiple emission sources. The M1 and M2 channels both show a southern footpoint located at $(920,-270)$ arcseconds. The presence of the second (northern) footpoint is also expected from the standard flare model. Although emission from the northern loop footpoint is tentatively observed in the M2 - and sometimes in the M1 - channel, centred at $(920,-220)$ arcsec, this emission is weak and not confidently observed. The reason for the weakness of the source may be explained by the main northern emission site being hidden behind the solar limb. Both high energy channels are also indicative of an emission source coming from above the loop top. This source may be associated with the Masuda source.

\section{Spatial correlation of time signals}

The identification of the observed mode requires the analysis of the spatial structure of the oscillation. An important characteristic of the mode is the phase relationship between the oscillation at different parts of the loop. 

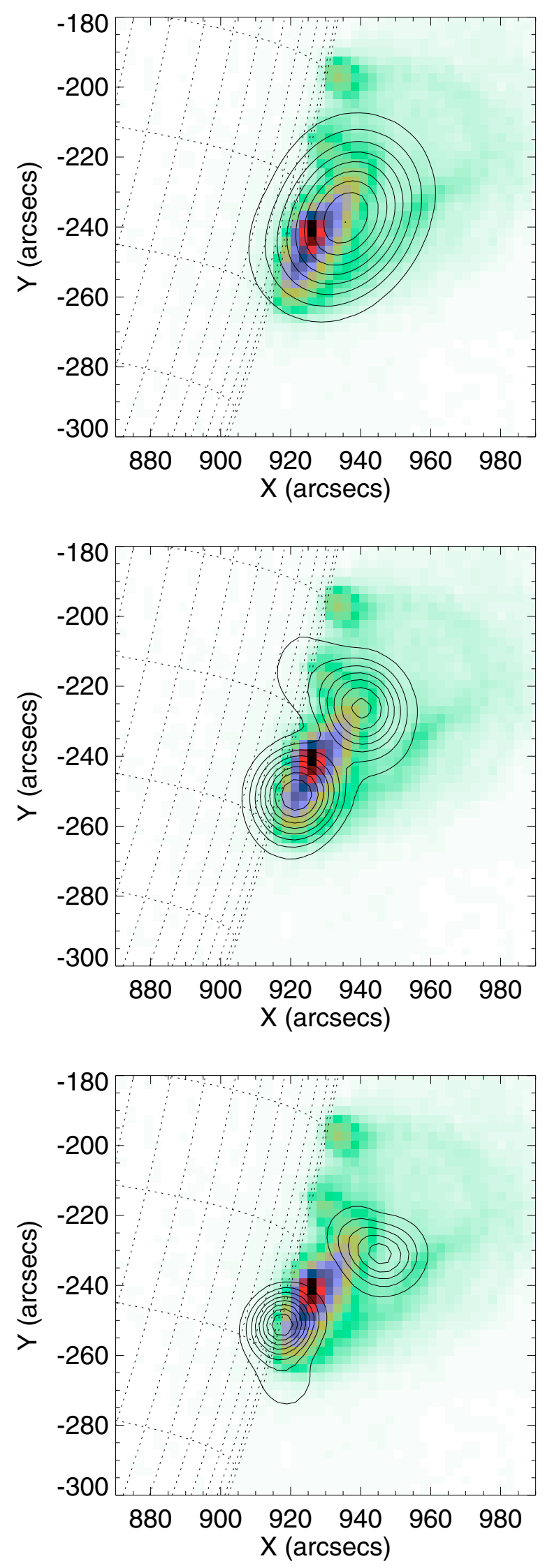

Fig. 5. Contour maps of hard X-ray emission overlayed on a soft X-ray image of the flaring loop at 01:57:50 UT, during the oscillatory phase. Top: the L (13-23 keV) channel, centre: the M1 (23-33 keV) channel, bottom: the M2 (33-53 keV) channel.

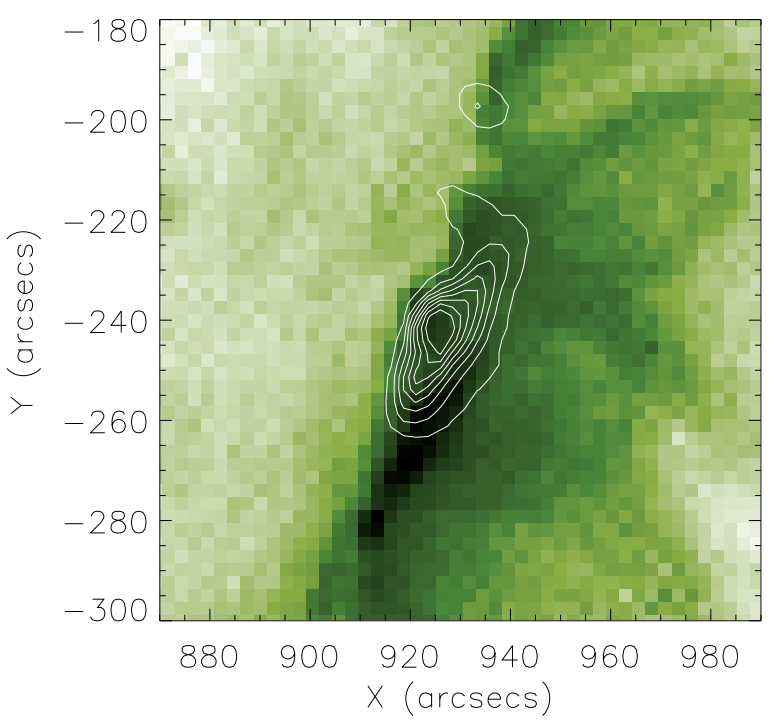

Fig. 6. Image: SOHO/EIT image of the flare of 8th May 1998. Contour: soft X-ray loop emission, obtained from the Yohkoh SXT.

\subsection{Periodmaps}

The application of the periodmapping technique (Nakariakov \& King 2007) allows us to determine the distribution of the dominating periods of time oscillations in the spectra of the individual pixels. As in this study we are interested in the mode with the period of about $16 \mathrm{~s}$ found in Sect. 2.1, we smooth the time signal of each pixel by 50 frames, which corresponds to $5 \mathrm{~s}$, suppressing the high frequency noise, and then subtract the signal smoothed by 500 frames, which corresponds to $50 \mathrm{~s}$. The latter operation removes the low frequency trend from the data. The periodmap of the data cube obtained in $17 \mathrm{GHz}$ is shown in Fig. 7. In the map, the colour of a pixel corresponds to the period of the highest peak in the spectrum of the time signal of the pixel, provided the spectral power of the peak is 5 times greater than the power averaged over the spectrum. Otherwise, the pixel colour is white. In addition, The pixels with the signals integrated over time, lower than $7.5 \times 10^{-3}$ of the integrated signal from the "strongest" pixel, are left blank. This operation allows us to subtract the spatial interference pattern which is the artefact of the image synthesis.

The periodmap shows that the period of about $16 \mathrm{~s}$ dominates in almost all pixels with time integrated signals stronger than the set threshold. Some pixels near the edge of the region of $16 \mathrm{~s}$ oscillations show the presence of higher periodicity. Thus, almost all segments of the flaring loop oscillate with the same period of about $16 \mathrm{~s}$.

\subsection{Correlation maps}

To investigate the spatial structure of phase of the oscillations, a cross-correlation analysis was used. First, a pixel with the master (reference) signal was selected, which we shall refer to as the "master" pixel. There is however more than one possible selection criterion for the master pixel. For example, the master pixel can be the pixel with the highest time variance of the signal. Alternatively it may be the pixel with the highest time-integrated intensity. Once the master pixel is selected, for each pixel of the image we calculate the correlation coefficient between the time signal of this pixel and the time signal of the master pixel for 


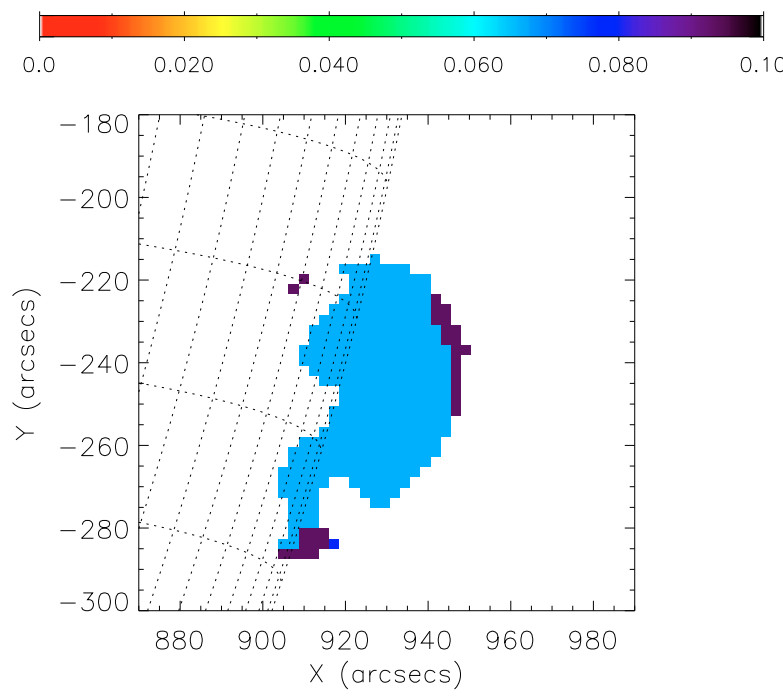

Fig. 7. Periodmap of the microwave emission at $17 \mathrm{GHz}$, obtained by the Nobeyama Radioheliograph during the pulsating regime of the limb flare of 8th May 1998. The bar shows the colour coding of the frequencies in $\mathrm{Hz}$.

different time lags between these signals. The correlation coefficient is given by:

$P_{x y}(L)=\left\{\begin{array}{l}\frac{\sum_{k=0}^{N-L-1}\left(x_{k+L}-\bar{x}\right)\left(y_{k}-\bar{y}\right)}{\sqrt{\left[\sum_{k=0}^{N-1}\left(x_{k}-\bar{x}\right)^{2}\right]\left[\sum_{k=0}^{N-1}\left(y_{k}-\bar{y}\right)^{2}\right]}} \text { for } \mathrm{L}<0 \\ \frac{\sum_{k=0}^{N-L-1}\left(x_{k}-\bar{x}\right)\left(y_{k+L}-\bar{y}\right)}{\sqrt{\left[\sum_{k=0}^{N-1}\left(x_{k}-\bar{x}\right)^{2}\right]\left[\sum_{k=0}^{N-1}\left(y_{k}-\bar{y}\right)^{2}\right]}} \text { for } \mathrm{L} \geq 0,\end{array}\right.$

where $x$ and $y$ are the two datasets, and $L$ is the time lag. Visualising the results obtained with this approach, one can make, e.g., either a map showing the spatial distribution of the correlation coefficient value calculated at a specified time lag, or a map showing the time lags corresponding to the highest values of the correlation coefficient for each pixel.

The top panel of Fig. 8 is a correlation power map of microwave emission, calculated with zero time lag $-P_{x y}(0)$. As the master pixel we choose the pixel with the maximum value of the time integrated intensity, found near the centre of the loop emission. Here, for clarity, we have restricted our attention to areas showing a correlation coefficient power of 0.5 or greater. Pixels with $P_{x y}$ less than 0.5 are blank. The strong correlation throughout the emitting region is evident. The map also reveals some evidence of a loop-like structure.

The bottom panel of Fig. 8 shows the spatial distribution of time lags between the time signal from the master pixel and other pixels, which correspond to maximum correlation coefficients. The emitting region appears to be strongly correlated in time, with a maximum shift in time of about $2 \mathrm{~s}$ occurring towards the edge of the region.

A cross-correlation analysis of the hard X-ray sources did not succeed in either the M1 or M2 bands. The northern footpoint source is too weak to be meaningfully analysed. This is unfortunate as we would expect the two footpoints to be well correlated in time.

\section{Flare evolution}

It is also possible to use the hard X-ray data to investigate the evolution of the flare emission.

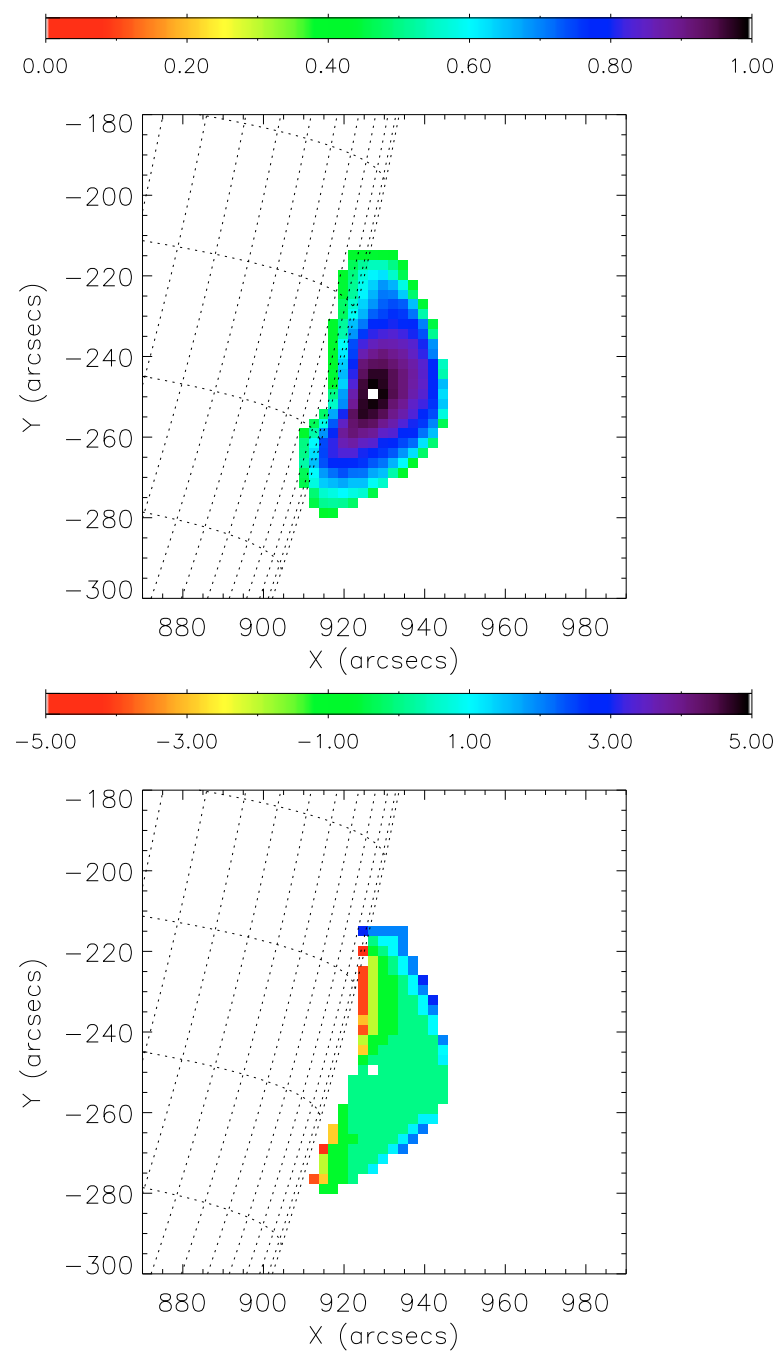

Fig. 8. Top: cross-correlation coefficient map for background subtracted microwave emission at $17 \mathrm{GHz}$, obtained by the Nobeyama Radioheliograph during the pulsating regime of the limb flare of 8th May 1998. The correlation coefficient is calculated with zero time lag. The master pixel is the pixel with the highest time-integrated intensity of the signal. The bar shows the colour coding of the correlation coefficient. Bottom: correlation time-lag map for microwave emission. The bar shows the colour coding of the time lag in seconds. In both panels the central white point corresponds to the master pixel.

The presence of a stable hard X-ray footpoint throughout the impulsive phase of the flaring emission is clearly illustrated by Fig. 9. The emission from the loop top is observed to shift in location and become more complex in structure over the same period. After the end of the impulsive phase - at 01:59 - the southern footpoint disappears, leaving only the loop top emission. This emission decays slowly over time, much like the thermal emission in the L (13-23 kev) energy channel.

Magnetograms of the active region prior to this event indicate the presence of a single sunspot, suggesting this event began with flux emergence leading to magnetic reconnection. Yohkoh SXT images also show a slight but steady outward expansion of the flaring loop over time, consistent with this interpretation.

\section{Discussion}

Quasi-periodic pulsations were clearly observed in both microwaves and hard X-rays for this event. It was also possible 

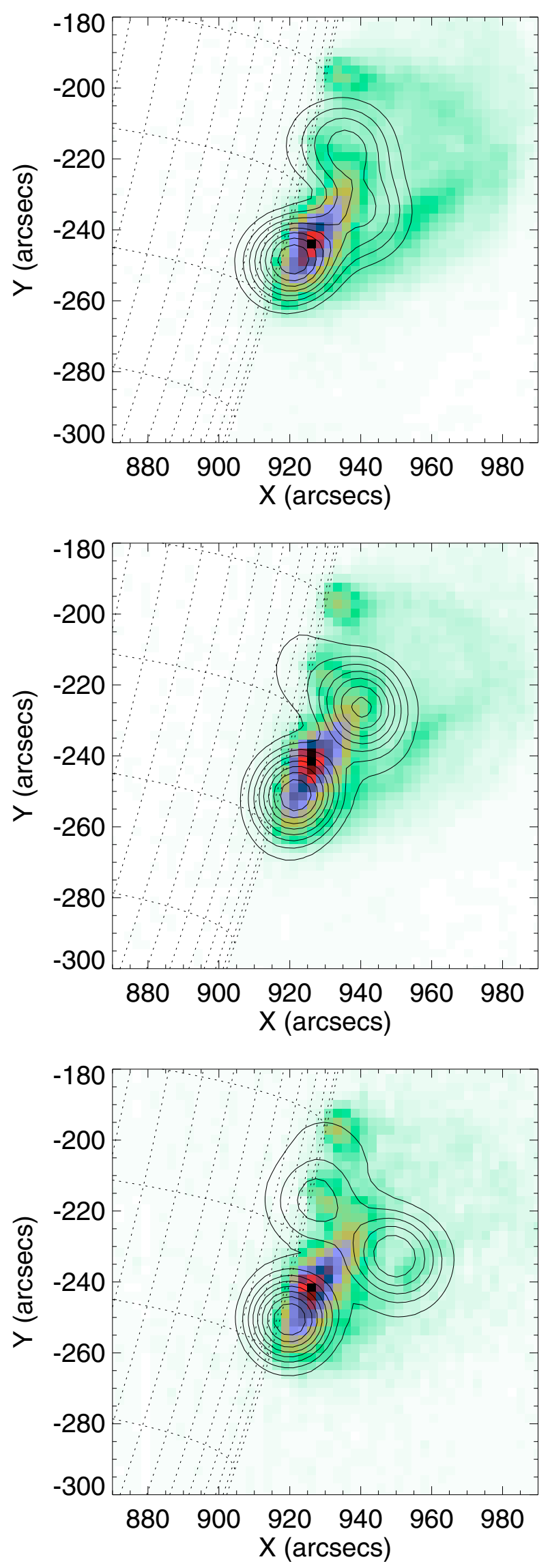

Fig. 9. Hard X-ray contours from the M1 (23-33 kev) channel overlayed on an SXT image, at 01:57:11, 01:57:49 and 01:58:28 respectively. to spatially resolve at least two different hard X-ray sources in the higher energy bands of the HXT, located at the southern loop footpoint and the loop top respectively. There is also some evidence of a hard X-ray source at the primary flare's northern footpoint. This is weak and only present at the onset of the impulsive phase. According to the standard flare model two footpoint sources are typical. We postulate therefore that the main emission site for the northern footpoint is hidden behind the limb of the sun.

In Stepanov et al. (2004) this event was considered as an MHD ballooning mode. However, more detailed analysis presented here does not support this interpretation. Periodmapping and the cross-correlation analysis of the microwave emission shows that well correlated oscillations with a period of about $16 \mathrm{~s}$ are distributed over the bulk of the loop. Also, the evidence of the characteristic ballooning mode "tongues" was not found in either X-ray or microwave emission. Thus, we conclude that the observed oscillation is caused by another reason.

We also exclude the interpretation of the observed quasiperiodic pulsations in terms of flare-induced slow magnetoacoustic waves (see, e.g. McAteer et al. (2005)). The observed emission is clearly connected with the non-thermal electrons. It is not clear how a slow wave which is mainly acoustic in nature could affect the dynamics of the non-thermal electrons and hence their precipitation rate. Slow waves can produce the variations of the microwave emission by modulation of the ratio of the plasma frequency to the electron gyro-frequency (see Nakariakov \& Melnikov 2006). However there are no known mechanisms which would link the modulation of the hard X-ray emission with slow waves. A similar problem takes place with interpretation of the observed oscillations of the hard X-ray emission in terms of a kink or torsional mode; they do not significantly affect the non-thermal electron dynamics.

On the other hand, the sausage mode can produce modulation of the microwave (Nakariakov et al. 2003) and hard X-ray (Zaitsev \& Stepanov 1982) emission. The period of the sausage mode is determined by the loop length. The loop length was estimated to be between $40-80 \mathrm{Mm}$. Unfortunately, due to the position of the flare on the limb measurement of the line-ofsight angle is difficult. Soft X-ray and EUV images indicate that there may be more than one loop in this region. In particular, there is some evidence of a second, cool loop above the primary loop. The northern footpoint of this is often visible at about $(930,-200)$ arcseconds, although it is not generally a source of hard X-ray emission. Additionally, magnetograms of the active region in the days preceding this event show only a single sunspot, suggesting that this flaring event was an emergent phenomenon. As a result a sunspot pair cannot be used as an alternative length estimate. However, the characteristic period of the oscillations, $16 \mathrm{~s}$, together with this rough length estimate, is consistent with the sausage mode interpretation (see Nakariakov et al. 2003). As the period of the global sausage mode - with the wavelength equal to double the loop length - is determined by ratio of the wavelength to the Alfvén speed outside the loop, this interpretation yields the estimation of the external Alfvén speed in the range from 5 to $10 \mathrm{Mm} / \mathrm{s}$.

An alternative interpretation of the observed quasi-periodic pulsations could be a periodic regime of magnetic reconnection. In this case, the acceleration of non-thermal electrons is periodically modulated, causing the periodic variation of the non-thermal electron density, and hence the periodic modulation of the microwave and hard X-ray emission. However, it is not clear what would determine the period of the oscillations in this case. One possibility is the periodic triggering of magnetic 
reconnection by an external oscillating loop (Nakariakov et al. 2006). The theory of this phenomenon needs to be developed. Hence, we cannot exclude this mechanism from consideration.

Useful additional information could be provided by spectroscopic instruments. Unfortunately, the spectral information available for this event is very limited. As mentioned previously, the Nobeyama Radioheliograph registers little above background noise at $34 \mathrm{GHz}$. Similarly, useful data is only available from the Nobeyama Radiopolarimeters at 3.75, 9, and $17 \mathrm{GHz}$. The strongest emission is in the $9 \mathrm{GHz}$ band, indicating that the gyrosynchotron turnover frequency is somewhere in this region.

Hard X-ray spectral information is equally limited, as the Yohkoh satellite observes in only four energy bands, the highest of which observes little above background levels. Therefore, no meaningful spectral analysis is possible.

\section{Conclusions}

A spatially resolved analysis of the solar flare of 8th May 1998 shows that it may be considered as a Masuda-type event, as evidenced by a hard X-ray emission source above the loop top. In high energy bands a strong southern footpoint is also observed. The absence of a noticeable northern footpoint in hard X-ray emission is attributed to the footpoint being obscured behind the solar limb. Magnetograms from the SOHO satellite provide evidence that this event was an emergent phenomenon leading to magnetic reconnection. Yohkoh SXT images also show an expansion of the loop over time.

The analysed flare has perhaps the most pronounced flaring QPP since the famous $8 \mathrm{~s}$ period oscillatory event observed on the 7th of June, 1980 (Kane et al. 1983) with ISEE-3 in X-rays and with the Nobeyama Radiopolarimeter in microwaves. In the event analysed here, the high quality $16 \mathrm{~s}$ periodicity is seen to be very stable during the flare, and was found to be present in the data with more than $99 \%$ confidence. In contrast with the event on 7th of June, 1980, this time spatial information is available, allowing us to determine the MHD mode responsible for the oscillation.

The observed period of $16 \mathrm{~s}$ together with an estimate of the loop length is consistent with the interpretation of the QPP in terms of the sausage mode which periodically changes the loop cross-section and the magnetic field. In this case the microwave emission is modulated by the periodic variation of the magnetic field in the emitting plasma. The periodic modulation of the hard X-ray emission is caused by the periodic modulation of the mirror ratio because of the variation of the loop cross-section. An alternative interpretation is the periodic modulation of the flaring energy release (either a periodic regime of magnetic reconnection, or periodic triggering of reconnection by an external waves), and hence the particle acceleration by some external oscillation. However, no evidence of such an oscillation was found in this event.

The observed period of $16 \mathrm{~s}$ is perhaps a typical period for the global sausage oscillations in flaring regions (e.g. with a similar value obtained in Nakariakov et al. 2003). This period is significantly shorter than the periods of quasi-periodic pulsations associated with slow magnetoacoustic modes (McAteer et al. 2005; Mitra-Kraev et al. 2005) and those possibly triggered by an external kink oscillation (Foullon et al. 2005). Confident discrimination between the flaring oscillations caused by global sausage modes and by oscillatory regimes of magnetic reconnection is impossible until the theory of the latter is understood.

Acknowledgements. The authors acknowledge helpful discussions with E. Verwichte, and are very grateful to V. Grechnev for the help with the preliminary analysis of this event. The work was supported by the Royal Society British-Russian Research Collaboration grant. ARI acknowledges the support of an STFC PhD studentship. VFM acknowledges the support of RFBR grants 06-02-39029, 06-02-16295, 07-02-01066.

\section{References}

Foullon, C., Verwichte, E., Nakariakov, V. M., \& Fletcher, L. 2005, A\&A, 440, L59

Grechnev, V. V., White, S. M., \& Kundu, M. R. 2003, ApJ, 588, 1163

Horne, J. H., \& Baliunas, S. L. 1986, ApJ, 302, 757

Kane, S. R., Kai, K., Kosugi, T., et al. 1983, ApJ, 271, 376

Masuda, S., Kosugi, T., Hara, H., Tsuneta, S., \& Ogawara, Y. 1994, Nature, 371, 495

Mathioudakis, M., Seiradakis, J. H., Williams, D. R., et al. 2003, A\&A, 403, 1101

McAteer, R. T. J., Gallagher, P. T., Brown, D. S., et al. 2005, ApJ, 620, 1101

Melnikov, V. F., Reznikova, V. E., Shibasaki, K., \& Nakariakov, V. M. 2005, A\&A, 439, 727

Mitra-Kraev, U., Harra, L. K., Williams, D. R., \& Kraev, E. 2005, A\&A, 436, 1041

Nakariakov, V. M. 2007, Adv. Space Res., 39, 1804

Nakariakov, V. M., \& King, D. B. 2007, Sol. Phys., 241, 397

Nakariakov, V. M., \& Melnikov, V. F. 2006, A\&A, 446, 1151

Nakariakov, V. M., Melnikov, V. F., \& Reznikova, V. E. 2003, A\&A, 412, L7

Nakariakov, V. M., Foullon, C., Verwichte, E., \& Young, N. P. 2006, A\&A, 452, 343

Scargle, J. D. 1982, ApJ, 263, 835

Stepanov, A. V., Kopylova, Y. G., Tsap, Y. T., et al. 2004, Astron. Lett., 30, 480

Tapping, K. F. 1983, Sol. Phys., 87, 177

Zaitsev, V. V., \& Stepanov, A. V. 1982, Soviet Astron. Lett., 8, 132 